\title{
Missing opportunities for preventing unwanted pregnancy: a qualitative study of emergency contraception
}

\author{
Pranitha Maharaj, Michael Rogan
}

School of Development Studies, University of KwaZulu-Natal, Durban, South Africa

\section{Correspondence to Prof Pranitha Maharaj, Associate Professor, School of Development Studies, University of KwaZulu-Natal, Durban 4041, South Africa; \\ Maharajp7@ukzn.ac.za}

Received 3 March 2010 Accepted 5 October 2010 towards EC in order to better understand factors depth ing uptake. The study drew upon 30 in public health facilities in Durban, South Africa. Results The results of the study highlight several barriers to the provision of EC in both public and private health facilities. The cost of EC products in commercial pharmacies is likely to be a major barrier to use for many women. In addition, providers in both public and private facilities are often reluctant to provide EC over the counter because they feel that the use of EC is likely to discourage regular use of contraception and increase the risk of unprotected sexual intercourse and, as a result, contribute to the spread of HIVIAIDS in South Africa. In addition, they reported that they do not have an opportunity to counsel women about EC because of time constraints. Providers in both the public and private sectors also demonstrated a level of uncertainty about the clinical effects of EC pills and on the regulations surrounding their provision. Discussion and conclusions Despite relatively progressive legislation on EC provision and the widespread availability of EC products in South Africa, providers in pharmacies, family planning clinics and public health clinics need more training on EC provision. Interventions should aim to educate health providers on both the clinical and social aspects of EC provision.

\section{Introduction}

In South Africa, the level of fertility among young women remains high. By the age of 18 years, one in five women have given

\section{Key message points}

Demand for emergency contraception (EC) is increasing, especially at pharmacies. However, cost remains a major barrier to access.

Providers expressed several reservations about the provision of EC over the counter, without a prescription.

There is a need for more training of providers on EC provision.

birth, and more than 30\% of women nationally have their first child before they attain the age of 20 years. ${ }^{12}$ Many births to mothers aged less than 20 years are unplanned and/or unwanted. ${ }^{23}$ Emergency contraception (EC) is known to be highly effective at preventing, or reducing the risk of, an unwanted pregnancy after unprotected sexual intercourse (UPSI). This includes all cases of incorrect use, method failure (such as condom slippage, leakage or breakage, missing hormonal pills or an intrauterine device expulsion) or failure to use contraceptives, including in cases of sexual assault. ${ }^{4} \mathrm{EC}$, also known as postcoital contraception or the 'morning-after' pill, has been shown to be effective for up to 120 hours (5 days) after UPSI. However, the efficacy of the contraceptive appears to decline with time. ${ }^{45}$ This makes ease of access to EC products a critical component of its effectiveness.

Legislation affecting access to EC is relatively progressive in South Africa. Since 2000, EC products have been rescheduled to allow for over-the-counter access without a doctor's prescription. There are two dedicated EC products available on the commercial market and, to a limited extent, in some public sector health facilities. In 1999, the first dedicated EC pill was introduced to the South African market in the form of an estrogen/progestogen product. In 2001, a levonorgestrel-only EC pill with an improved side effect profile was made available. ${ }^{6}$ In public sector health 
facilities, EC products are available at no cost to the client and are usually provided in the form of 'cut-up' (i.e. repackaged) regular combined oral contraceptives (COCs). In commercial pharmacies, the levonorgestrelonly product (the most expensive option), the estrogen/ progestogen product (although recently discontinued) and repackaged COCs are all available as EC options.

In the years since EC has been available over the counter without a doctor's prescription, several studies have begun to investigate the role of health providers in facilitating access to EC. ${ }^{6-8} \mathrm{~A}$ recent review of EC studies in South Africa found that of the studies that have focused on the knowledge, attitudes and practices of providers, only two have included commercial pharmacists. ${ }^{9}$ One of these studies was conducted to examine pharmacists' knowledge and perceptions of EC pills in two urban areas in South Africa 2 years after the method became available over the counter without a prescription. ${ }^{7}$ The other study was published shortly after EC products were deregulated and prior to the widespread availability of the most recent EC product (the levonorgestrel-only pill). ${ }^{8}$

Despite the relative ease of access to EC products in South Africa, most studies suggest that use is low. ${ }^{3} 1011$ Increasing evidence suggests that it is not simply lack of knowledge that results in underutilisation of EC but also the attitudes of health providers and their reluctance to provide it. ${ }^{67} \mathrm{~A}$ study of adolescent mothers in South Africa found that the attitudes of providers play a critical role in facilitating access to EC. ${ }^{12}$ Provider knowledge and acceptance of EC constitute a crucial link to improving access to women who need it. ${ }^{12} 13$ Some argue that the promotion of EC by health providers is likely to lead to greater client awareness of the method and also increased availability. ${ }^{13}$ In addition, health professionals have the opportunity of counselling EC clients on other aspects of reproductive health such as sexually transmitted infections and HIV/AIDS. ${ }^{14}$ However, a study of two urban health facilities in South Africa observed that the quality of contraceptive counselling was variable and that there appeared to be little discussion of EC. ${ }^{15}$

Given the large number of unwanted pregnancies that occur every year in South Africa, as in many other parts of the world, as a result of failure to use contraceptive methods, sexual assault and incorrect use of barrier methods, EC has a crucial role to play in giving women an option to avoid the negative consequences of unwanted pregnancy, including the need for an abortion. ${ }^{4}$ The available evidence suggests that health providers may impede access to EC products. However, qualitative work that considers the perspectives of a wide range of providers is still needed, especially since there has been little work directed towards them since the deregulation of EC products. The aim of the study, therefore, was to investigate health provider knowledge and attitudes towards EC as a possible factor influencing uptake in South Africa.

\section{Methods}

The study drew upon qualitative data derived from in-depth interviews with health providers. In total, 30 in-depth interviews were conducted with providers at private clinics, commercial pharmacies, non-governmental family planning clinics and public health clinics in the city of Durban, KwaZulu-Natal, a large metropolitan area on the east coast of South Africa.

The sampling frame was restricted to the central district of Durban, as past studies have suggested that EC awareness and use is considerably higher in urban areas compared with rural areas in South Africa. ${ }^{616}$ As described elsewhere, ${ }^{17}$ this site was chosen because it serves as a major commercial centre in KwaZulu-Natal, South Africa and has the greatest range of EC products available. Past studies investigating EC in South Africa have reported difficulties in identifying providers and users of EC. ${ }^{10}$ The study area was therefore selected to ensure a high response rate among providers of EC since use is likely to be considerably higher in the sample area than in other parts of South Africa. As a result, the sample may not be representative of all health providers in Durban or to the rest of KwaZulu-Natal or South Africa in general.

In order to compile the sampling frame, a list of all pharmacies and dispensaries registered with the Pharmacy Council of South Africa was obtained and contact was made with the local and provincial health departments. The final list comprised a total of 53 pharmacies, dispensaries and clinics contained within the sample area. Of these facilities, only eight were public health facilities; the remainder were commercial pharmacies or private health clinics. Some of these were excluded from the study either because they were no longer in business $(n=8)$ or because they were wholesale suppliers $(n=5)$ and did not provide EC pills directly to the public. In addition, four commercial pharmacies were excluded because they did not sell EC products and therefore did not wish to participate in the study. The refusal rate among pharmacists that provide EC was relatively low; only six refusals were received and these were all due to time constraints. In the end, interviews were conducted with a total of 30 health providers. These comprised 20 retail pharmacists, two health workers from nongovernmental organisation-operated family planning clinics, six nurses from public sector comprehensive clinics, and two nurses from public sector family planning clinics.

Prior to the interviews, permission was obtained from the relevant authorities. It was easier to gain access to the private facilities because permission was usually obtained from the owners or managers of the facility. Access to the public facilities required obtaining permission not only from the local and provincial ministry of health but also the senior management at the facility. Informed consent was obtained from all the participating health providers prior to the interview. 
The interviews collected detailed information from providers including their background characteristics, knowledge of and attitudes to EC, and some of the factors facilitating and/or inhibiting use of EC among clients. All respondents were assured that their responses would be kept strictly confidential and the study would maintain their anonymity. An attempt was also made to ensure maximum privacy during the interview. Each interview lasted approximately 30-45 minutes. All the interviews were recorded with the permission of the respondent. Notes were also taken during the interview. The recorded interviews were transcribed and analysed using thematic analysis, which involves categorising the data according to salient themes. Themes are defined as the recurrent ideas or topics that are detected in the material being analysed and usually come up on more than one occasion in a particular set of data. ${ }^{18}$ The transcripts are used to illustrate particular findings from the in-depth interviews with providers.

\section{Results}

\section{Sample description}

Among the participating health providers there was a slightly higher representation of females than males. The average respondent was 40 years old and had 6 years of work experience in the health facility where the interview took place. Respondents in the commercial pharmacies were qualified pharmacists and health providers in all other facilities were registered nurses. All of the providers reported that they had heard of EC and most (87\%) had received some training on it. In most cases (92\%) the providers received information on EC as part of their training on family planning methods. EC is still a relatively recent component of family planning, and as a result it may not have been a part of the training of all providers. Some of the providers had received their training more than 10 years ago. However, a few providers reported that they had received training about the product at their workplace, usually from the manufacturers of one of the EC products.

\section{Availability of EC}

All of the providers interviewed had heard of EC or the morning-after-pill and they were aware that it is effective in reducing the risk of pregnancy after UPSI. Providers seemed to have relatively good knowledge about when to recommend EC. Most stated that although an EC regimen may be started up to 72 hours after UPSI, it should preferably be taken within the first 24 hours following UPSI. Only a few providers knew that EC may be used up to 5 days after UPSI. Many of the interviewees also stressed that EC pills should not be used as regular contraceptives.

"We only give them in circumstances of unprotected intercourse if it happened within the last 72 hours. We provide ECs in the case of rape or a burst condom. We do not provide it if a person is not using a method of contraception and they say 'Can you give it to me I am going to have unprotected sex tomorrow?' No, we do not give it in that case." [Commercial pharmacist]

The choice of EC products appears to be fairly limited, particularly in public sector facilities. In public health clinics, EC is provided almost exclusively in the form of 'cut-up' regular COCs. Very few public sector health providers were even aware that a dedicated EC product was available in South Africa. In private pharmacies, both COCs and the dedicated levonorgestrel-only product are available to clients. In private facilities, providers argue that despite the improved side effect profile of the dedicated product, there is a higher demand for 'cut up' regular COCs than for dedicated EC products. Cost is a major factor influencing choice of EC products.

"The price of the product is the main consideration at the moment. Almost 99\% of the time people have already used the 'cut-ups' or their friends have used it and they come and ask for it by name. The costs are much lower for 'cut-ups'." [Commercial pharmacist]

The costs of dedicated EC products are considerably higher but they require a single dose, and according to the providers are associated with fewer side effects. In addition, providers in private facilities suggest that compliance is higher with dedicated products. The use of non-dedicated products requires the administration of two doses within 120 hours of UPSI, with a 12-hour interval between doses. Pharmacists observed that this reduced the effectiveness because some clients do not adhere to the instructions.

"People have problems with regular combined oral contraceptives because there are multiple doses. Clients have to take the second dose after 12 hours and not all of them are compliant. We have had cases where people had lost the second dose or the box." [Commercial pharmacist]

Providers in public health facilities report that demand for EC products is very low and that this is likely related to the inability to offer walk-in EC services. In most public facilities there is a long waiting period, which may discourage use of EC products. Clients often have to wait in long queues before they receive EC.

"It is very rare to find a client requesting EC. We usually give it to the client who reports having unprotected sex: either the condom burst or she was not using any other contraceptive method." [Public health provider]

Conversely, providers in private facilities report that requests for $\mathrm{EC}$ are common and on the rise, especially among young women aged between 18 and 30 years. They noted that women are more likely to request EC but there is also a growing demand among male partners. [NB. This finding is supported by results from South Africa's most recent Demographic and Health Survey, which suggests that men in the age group 15-59 years report higher levels of 'ever use' of emergency 
contraception (1.4\% of men aged $15-59$ years reported ever using EC compared with just $0.5 \%$ of women aged $15-49$ years). $]^{3}$ Older women are more likely to be using a long-term contraceptive method, and according to the providers they sometimes request $\mathrm{EC}$ if they experience method failure but, in general, are not typical EC clients. As one public health provider explained:

"When you are in your 30s you are usually more mature. You are usually more stable in your job and you know that you cannot afford to fall pregnant because of the financial implications. So you are either on a contraceptive already or you have taken the necessary precautions. I think I may see only one or two a year in that age group." [Public health provider]

\section{Perceptions of EC}

In general, providers, particularly in public sector facilities, were in favour of improving the availability of EC services and were aware of the potential benefits of EC in preventing unplanned and often unwanted pregnancies. However, most providers expressed misgivings about the use of EC. Moreover, the conditions under which providers were willing to supply EC were often narrowly defined, and none of the providers in the private or public health facilities reported supplying an advance dosage for clients.

"I do not have a problem with EC for the simple reason that I would rather cope with one mistake than two. I would rather she came here not having used a method and wanting EC than the client having an unwanted pregnancy; because that is likely to lead to problems. Otherwise we are going to have mega social problems which we are already encountering now. So to avert that possibility I have no hesitation in providing EC.... [But] I do not believe clients should be given an advance dose of EC. I think they should come to the clinic when they need it because you get an opportunity to counsel them." [Public health provider]

Almost one-third of providers expressed concern that the increased availability of EC might impact negatively on clients' behaviour. They were particularly concerned that increased accessibility of EC will lead to an increase in risky sexual behaviour or a reduction in the use of other (particularly barrier) methods of contraception. This was especially worrying to providers in view of the high rate of HIV/AIDS infections in South Africa. Some providers, for example, commented:

"Obviously EC encourages risky behavior. When they come in to request EC it is clear that they are having unprotected sex, and in South Africa unprotected sex is a risk because of the high levels of HIV infection." [Commercial pharmacist]

"It is good that you cannot advertise EC products. If everybody knew that this is available you tempt them and obviously sales will go up but there will be an increase in sexually transmitted diseases." [Commercial pharmacist] "I do not feel uncomfortable giving it but it concerns me that there is such a high demand only because of the high levels of HIV infections. What is particularly worrying is that the demand is great among young people aged 18-25 who are relatively educated about HIV/AIDS. So it concerns me that despite the education, there is not the slightest fear about the possibility of contracting HIV." [Commercial pharmacist]

One provider at a commercial pharmacy remarked that the facility only offered dedicated EC products because "we try to discourage people from using it. We use the high costs [of the dedicated product] as a means of preventing people from using it”. Conversely, several providers in the public sector felt that EC use was low because the option of termination of pregnancy is more popular, particularly among young women. As one public health provider argued:

"I think, as I have said, they are well aware of EC now, but I think that there are other options that they can use, like termination. It is very rare that they think about it now because they know that there is another option if they do happen to fall pregnant. There is always termination. So they do not stress about it. The teenagers especially do not stress about it. This is why I think EC use is very rare because abortion is one big option." [Public health provider]

A few providers expressed uncertainty about EC and were not entirely comfortable with providing EC but they felt that they were not able to change the situation. This attitude was captured by one provider in a public facility who stated that: "At the end of the day we are here to offer a service. The client has come for a service and it is their right to receive it". In South Africa, health providers are required by legislation to provide EC without restrictions. However, most providers in the private facilities reported that they do not usually counsel clients about the role of EC in preventing unwanted pregnancy after UPSI because of the high client load. When clients request EC the providers give it to them but often do not voluntarily offer them additional information about it. However, providers reported that it was not possible to restrict frequent use of EC because of their high client load.

"I wish I could restrict the number of times that EC is prescribed to an individual, but in terms of the current circumstances it is not possible. The pharmacy is too busy and most often we do not always remember the people who request EC. And we often do not have time to take down a profile.” [Commercial pharmacist]

A few providers also expressed concerns about the side effects of EC. They felt that inappropriate or frequent use of EC may lead to health problems. One pharmacist incorrectly stated that multiple use of EC increases the risk of cancer, and as a result was reluctant to provide clients with repeat dosages. Others were concerned about the impact of EC on hormone levels.

"If you are using EC on a continuous basis I think it poses a health risk. I can provide counselling to a woman in one week but in the next week she can go to another pharmacy where the pharmacist will not know her and counsels her 
as well. The next week she can go to another pharmacy to get her supply of EC. This can throw her whole cycle into complete disarray and increase the risk of cancer." [Commercial pharmacist]

A few providers observed that they would prefer to advocate EC rather than termination of pregnancy. Although most providers knew that EC does not cause abortion, a few perceived that EC may be a form of abortion or may cause harm to a fetus if taken too late.

"I am on the fence about it. I do it, but at the back of my mind I am thinking 'Is this person pregnant? I hope that I am not causing any adverse effects to the fetus'. However, I would rather issue EC than perform termination." [Commercial pharmacist]

In some cases, providers also reported refusing to supply EC to clients, often as a result of uncertainty about the age at which a client can purchase EC products without a guardian's consent. They reported that they often referred young men and women to the clinic for more effective methods of contraception. In two cases, providers were unaware that EC could be provided without a doctor's prescription and as a result regularly refused provision. Perhaps the largest degree of uncertainty among providers, however, was around the age of clients that request EC. In fact, the majority of providers in both the public and private health facilities expressed great concern about supplying young people, in particular, with EC. They often used age restrictions to limit EC use among this stratum of the population.

"I feel uncomfortable about prescribing it to a young person who is under 16 years but I try to separate my personal and professional view. They need to be older than 18 for me to accept that they can make their own decision in their own capacity. If they are younger I usually refer them to a clinic or somebody that can give consent but most of the time it is impossible for them to get consent, because of stigma. Most people do not want to go to the clinic if it is a small community. Apparently people are uncomfortable with the lack of privacy in these clinics and that all people are known in these communities and they do not want to be stigmatised." [Commercial pharmacist]

\section{Barriers to EC use}

Despite their concerns with providing EC, health providers were able to identify a number of barriers that EC users are likely face in South Africa. Many of the pharmacists felt that young people do not receive sufficient information about EC and contraception, more generally, and they argued that there is a need for programmes to create greater awareness of the correct use of EC, particularly in rural areas. Many people have heard of EC but they lack detailed information about the method, which is illustrated by the comment of one provider: "Most people have heard of it but they may not know how long after unprotected sex they should use it”. In rural areas, access to EC is limited and clients are often not given a full range of contraceptive options. In addition, EC cannot be advertised and, according to many providers, this is a major factor that is limiting awareness of the product in both urban and rural areas.

Many of the providers in the public health facilities felt that they were ideally placed to promote EC. However, the heavy client load and the severe shortage of trained staff serve as an obstacle to the promotion of EC. Many providers stated that the long queues at public health facilities discourage them from counselling clients about the risks associated with UPSI.

\section{"Ideally I should be going into details and saying that: 'These are the methods that we have available. This is what you should be on. If you have unprotected sex this is what should be done'. However, sometimes we do not have the time. In most cases, we do not have time to provide detailed information and education because of staff short- ages and increased workload." [Public health provider]}

Providers at public facilities reported that clients are more likely to obtain EC from pharmacies than public clinics. It is easier to obtain EC from pharmacies because clients do not have to queue for a long period of time. However, clients have to pay for EC products that they obtain at pharmacies and they do not receive information about the product. In contrast to the view that heavy workloads and staff shortages prevent public sector health providers from counselling EC clients, one public health provider outlined the advantage of EC provision in the public sector as being a source of information.

"A lot of clients will come here because they get information from us. We counsel clients and help them make an informed decision whereas at the pharmacies it is a matter of 'here is my money' and 'here is the product'. I think that clients appreciate the services they receive from us. They know that they are going to encounter side effects - they can cope with them because we have explained the side effects. They more or less know what to expect and are comfortable with it." [Public health provider]

Conversely, the consultation with the provider, particularly in pharmacies, is often very short, lasting only a few minutes and not allowing sufficient time to counsel clients about the benefits of EC. As a result, some providers tend to give clients a fact sheet which contains all the relevant information about the product. On the whole, in both the private and public sectors, providers are faced with heavy client loads, which makes it difficult for them to give each client personal attention. Clients rarely have an opportunity to ask any questions about their concerns. In addition, some clients are embarrassed to be seen obtaining EC. Sometimes the lack of privacy may affect clients' willingness to provide sensitive information. Other clients may listen in on the consultation and clients in the consultation may feel uncomfortable revealing the real reason for visiting the clinic. 
"Consultations last approximately one minute. Most [clients] do not have the time. They do not want to listen to a whole long story. They want the stuff and they want to leave. And some of them are embarrassed." [Commercial pharmacist]

Some of the providers also reported that they would like to receive more information on $\mathrm{EC}$ in order to improve the client-provider relationship. However, providers may be constrained in the advice that they can offer by their lack of training. Some providers had completed their training prior to the availability of EC products in South Africa. As a result they felt that there was an urgent need for refresher training to update providers on new contraceptive technologies. Some providers also felt that they would like to receive more information about the side effects of EC.

"As a pharmacist there is not much emphasis put on EC, and these new contraceptive methods. I qualified before EC was introduced. The mechanisms of it are, I would not say sketchy, but I would like to receive more information on it." [Commercial pharmacist]

\section{Discussion}

As part of a larger study investigating the context of EC provision and use in South Africa, this paper offers several contributions to the existing literature on EC in South Africa. First, the study is unique in that, to the best of the authors' knowledge, it is the only EC study in South Africa to conduct research with commercial pharmacists, public sector health providers, and specialised family planning providers. Second, while the results cannot claim to be generalisable, the strength of the paper lies in the qualitative design that offers some valuable insights into factors influencing EC use by drawing upon in-depth interviews with a wide range of EC providers. Third, the study gathers recent data from health providers in a site in which EC provision is likely to be relatively common compared with other parts of the country. Finally, the study offers insight into the provision of EC nearly 10 years after both the introduction of a dedicated EC pill in South Africa and the passing of legislation making EC products available without a doctor's prescription.

There are some indications that there is an increasing demand for EC, especially at private facilities. Pharmacies remain the key access point for EC products and, tellingly, product price is a main consideration for many EC clients. As in other local studies, ${ }^{6} 1011$ EC use in public sector clinics and facilities was found to be very low, despite the fact that the majority of South African women rely on the public health sector for their contraceptive supplies. The greater accessibility of EC at pharmacies notwithstanding, the cost of EC products in pharmacies is likely to be prohibitive for many women, and the fact that some pharmacists reported using the higher cost of the dedicated product as an access barrier is a major concern. In both commercial pharmacies and public health clinics, consultation time was reported to be a constraint to adequate counselling of EC clients. Almost all providers in the pharmacies complained of not having time to counsel EC clients and the great majority of pharmacies did not have a private counselling room in the facility.

Long waiting times for consultation at public clinics is another likely reason for the low uptake of EC in the public health sector. Long waiting times have been shown to discourage potential clients from seeking services. ${ }^{19}$ This is likely to be particularly important for potential EC clients as timely access is an important component of the treatment. Moreover, some providers may be constrained in the advice they can offer by their lack of adequate training. As a result, they are unable to offer clients a comprehensive range of services. Moreover, the large client loads make it difficult for providers to offer additional information to clients. Under the pressure of a long queue, it is hardly surprising that most providers deal as efficiently and quickly as possible with patients' explicit needs before moving to the next patient.

Both pharmacists and public health workers expressed a number of concerns with supplying EC, with the most common perhaps being that repeat use of EC might be harmful to women and could discourage regular contraception use. This particular theme has also been noted in the international literature on EC despite the fact that no studies have found a direct link between EC use and regular UPSI. ${ }^{20} 21$ Indeed, one European study even found that EC use was the catalyst to adopting more regular forms of contraception. $^{22}$ Despite the evidence demonstrating that EC is clinically safe, is not linked with cancer $^{23}$ and has no adverse effects, the 'gate-keeping' of EC products is a relatively common practice among the providers participating in the study. Some of the main reasons given for limiting or restricting access to EC products included: fears of repeat use by clients, the safety of EC products, uncertainty regarding the regulations surrounding EC, a feeling that EC use encourages promiscuity, and a reluctance to provide EC to younger women. The fact that age was a concern for a number of pharmacists and public health providers, despite the fact that the number of unplanned and sometimes unwanted pregnancies is high among young women, is particularly worrying.

One of the key findings of the study is that providers in pharmacies, family planning clinics and public health clinics need more training on EC provision. In addition to the clinical information that providers themselves indicated that they would like to have, the growing number of studies that have shown that EC use does not discourage regular contraception use and does not lead to risky sexual behaviour should also be communicated to providers. ${ }^{202224}$ The present study found that many providers felt that EC promoted promiscuity and UPSI. As such, many of the narratives presented in this paper demonstrate the palpable 
uncertainty that many providers have in providing EC to their clients, despite its recent deregulation. Some of the reservations about EC are also related to concerns about the risk of UPSI in the context of a high HIV prevalence in KwaZulu-Natal.

It is important to note some of the limitations of this study. The findings of the study may have limited generalisability to other populations because of the small sample size. Moreover, the study site was specifically chosen in order to facilitate access to health providers where EC is most likely to be supplied. It is not unlikely that health providers who did not participate in the study may be less likely to provide EC. Interpretation of the findings is also limited by provider's self-reports. It is also possible that social desirability may have altered the reporting of attitudes towards EC to some extent. As in other studies, ${ }^{10}$ providers in public health facilities reported supplying EC only in very rare cases and only perhaps once or twice a year. As a result, recall bias may be a particular problem among these health providers. In terms of the limitations associated with interviewing commercial pharmacists, difficulties such as a lack of privacy, frequent interruptions and some degree of suspicion towards the research process all may have compromised the findings to a certain degree. Some of these challenges involved with researching EC access in pharmacies have also been documented in the international literature. ${ }^{25}$

Overall, however, the results of the present study suggest that much more emphasis should be placed on obtaining provider support for EC. This is likely to be an important lesson in other contexts where EC is available over the counter without a prescription. The findings presented here suggest that the availability of dedicated EC products and access to EC pills without a doctor's prescription are not enough. Health providers need to be able not only to provide EC, but they must be comfortable enough to promote the use of EC, particularly among those at high risk of an unwanted pregnancy. The adequate training of health care providers, both within and outside the public health sector, is therefore an essential step in improving EC uptake. The fact that the majority of providers in this study expressed a strong interest in obtaining more information about EC is encouraging in this regard.

\section{Competing interests None.}

Ethical approval Ethical approval for the study was obtained from the university committee at the University of KwaZulu-Natal.

Provenance and peer review Not commissioned; externally peer reviewed.

\section{References}

1 Department of Health of South Africa and Macro International. South Africa Demographic and Health Survey 1998. Pretoria, South Africa, and Calverton, MD: Medical Research Council and Macro International, 1999.
2 National Research Council and Institute of Medicine of the National Academies (NRC-IOM). Growing Up Global: The Changing Transitions to Adulthood in Developing Countries. Lloyd CB (ed.). Washington, DC: The National Academies Press, 2005.

3 Department of Health of South Africa and Macro International. South Africa Demographic and Health Survey 2003. Pretoria, South Africa, and Calverton, MD: Medical Research Council and Macro International, 2003.

4 Westley E, von Hertzen H, Faundes A. Expanding access to emergency contraception. Int J Gynaecol Obstet 2007;97:235-237.

5 Cheng I, Gulmezoglu A, Van Oel CJ, et al. Interventions for emergency contraception. Cochrane Database Syst Rev 2004;3:CD001324.

6 McFadyen L, Smit J, Mqhayi MM, et al. Expanding Contraceptive Choice: An African Study of Emergency Contraception. A Multi-centre Situational Analysis of Emergency Contraception Provision and Utilization at Public Sector Clinics in South Africa. Johannesburg, South Africa: Reproductive Health Research Unit, 2003.

7 Blanchard K, Harrison T, Sello M. Pharmacists' knowledge and perceptions of emergency contraceptive pills in Soweto and the Johannesburg Central Business District, South Africa. Int Fam Plan Perspect 2005;31:172-178.

8 Hariparsad N. Knowledge of emergency contraception among pharmacists and doctors in Durban, South Africa. Eur J Contracept Reprod Health Care 2001;6:21-26.

9 Maharaj P, Rogan M. Emergency contraception in South Africa: a literature review. Eur J Contracept Reprod Health Care 2008;13:351-361.

10 Smit J, McFadyen L, Beksinska M, et al. Emergency contraception in South Africa: knowledge, attitudes, and use among public sector primary healthcare clients. Contraception 2001;64:333-337.

11 Myer L, Mlobeli R, Cooper D, et al. Knowledge and use of emergency contraception among women in the Western Cape province of South Africa: a cross-sectional study. BMC Womens Health 2007;7:14.

12 Ehlers VJ. Adolescent mothers' utilization of contraceptive services in South Africa. Int Nurs Rev 2003;50:229-241.

13 Delbanco SF, Mauldon J, Smith MD. Little knowledge and limited practice: emergency contraceptive pills, the public, and the obstetrician-gynecologist. Obstet Gynecol 1997;89:1006-1011.

14 Harrison J, Varnam M, Cardy G. A request for the 'morningafter' pill. Practitioner 1989;233:899-902.

15 Cooper D, Bracken H, Myer L, et al. Reproductive Intentions and Choices Among HIV-infected Individuals in Cape Town, South Africa. Policy Briefs. Cape Town, South Africa: Women's Health Research Unit, 2005.

16 Mqhayi MM, Smit JA, McFadyen ML, et al. Missed opportunities: emergency contraception utilisation by young south African women. Afr J Reprod Health 2004;8:137-144.

17 Rogan M, Nanda P, Maharaj P. Promoting and prioritizing reproductive health commodities: understanding the emergency contraception value chain in South Africa. Afr J Reprod Health 2010;14:9-20.

18 Hayes N. Framework for Qualitative Analysis in Doing Psychological Research. Buckingham, UK: Open University Press, 2000.

19 Huntington D, Schuler SR. The simulated client method: evaluating client-provider interactions in family planning clinics. Stud Fam Plann 1993;24:187-193. 
20 Anderson C, Blenkinsopp A. Community pharmacy supply of emergency hormonal contraception: a structured literature review of international evidence. Hum Reprod 2006;21: 272-284.

21 Polis CB, Schaffer K, Blanchard K, et al. Advance provision of emergency contraception for pregnancy prevention: a metaanalysis. Obstet Gynecol 2007;110:1379-1388.

22 Gainer E, Blum J, Toverud EL, et al. Bringing emergency contraception over the counter: experiences of nonprescription users in France, Norway, Sweden and Portugal. Contraception 2003;68:117-124.
23 Westley E, Glasier A. Emergency contraception: dispelling the myths and misperceptions. Bull World Health Organ 2010;88:243-244.

24 Raine TR, Harper CC, Rocca CH, et al. Direct access to emergency contraception through pharmacies and effect on unintended pregnancy and STIs: a randomized controlled trial. JAMA 2005;293:54-62.

25 Black K, Anderson C, Kubba A, et al. Involving pharmacists in sexual health research: experience from an emergency contraception study. J Fam Plann Reprod Health Care 2009;35:41-43. 